\title{
Comunicación

\section{Cultura migratoria y comunicación masiva e interpersonal en los imaginarios juveniles ${ }^{1}$}

Culture of migration and media and interpersonal communication

in youth's imaginaries

\section{MARTÍN ECHEVERRÍA VICTORIA²}

A partir de una propuesta acerca de la participación central de la comunicación interpersonal y masiva en la cultura de la migración, mediante los imaginarios que aquella produce, presentamos datos empíricos de jóvenes migrantes potenciales de una comunidad de Yucatán que demuestran que las noticias y las narrativas socializadas y apropiadas colectivamente, en efecto contribuyen a persuadir o disuadir a los sujetos a emigrar.

PALABRAS CLAVE: Cultura, migración, comunicación, imaginarios, jóvenes.
From a theoretical approach that emphasize the role of interpersonal and media communication in the culture of migration through imaginaries, we present empirical data from young potential migrants of a community of Yucatan, that demonstrates that news and narratives socialized in the community indeed persuades or deters subjects to migrate.

KEY WORDS: Culture, migration, communication, imaginaries, youth.

1 El artículo se desprende del proyecto CONACYT/FOMIX "El impacto económico y sociocultural de la migración internacional en el municipio de Tunkás: consecuencias económicas y culturales y su reproducción en la etnia maya" (clave 108867).

2 Universidad Anáhuac Mayab, México.

Correo electrónico: echevemartin@yahoo.com.mx

Carretera Mérida Progreso Km. 15.5 AP. 96, Cordemex, C.P. 97310; Mérida, Yucatán, México. 


\section{INTRODUCCIÓN}

Con el fin de explicar motivaciones migratorias que no responden del todo a factores económicos o sociales, los académicos de la migración proponen la existencia de una cultura de la migración que en el nivel comunitario proporciona incentivos para migrar en forma de prestigio, estatus, valores asociados al cuidado de la familia y de manera importante el estilo de vida que supuestamente faculta la migración hacia otros países. Como un objeto remoto y difuso, dicho estilo de vida evidentemente solo puede ser contemplado de forma imaginaria por los migrantes potenciales.

Este imaginario depende estrechamente de la información que es introducida a la comunidad en forma de narrativas a través de canales de comunicación interpersonales o mediáticos. Es aquí donde la disciplina de la comunicación pudiera tener un papel relevante para describir y explicar tanto la conformación de dichos imaginarios y la manera en que una cultura de la migración prevalece o se transforma con la participación de ellos, como los procesos de reducción de incertidumbre facilitados por la información acerca del destino y tránsito migratorio. Esta dimensión "comunicativa" es sumamente relevante porque la evaluación de la conveniencia del destino migratorio toma como criterio fundamental las gratificaciones y beneficios imaginados por los migrantes potenciales acerca del destino migratorio, así como la ausencia de riesgos al tratar de conseguirlos.

El presente artículo elabora una propuesta analítica para introducir la perspectiva de la información y la comunicación al estudio de las comunidades rurales expulsoras de migrantes y proponer hipótesis acerca de la forma en que la comunicación interpersonal, la mediática y los bienes simbólicos, como vectores de significados, perfilan la cultura de la migración de una determinada comunidad. Para reforzar nuestra propuesta reportamos resultados de un estudio empírico realizado en la comunidad de Tunkás, Yucatán, escenario de un flujo migratorio internacional robusto y antiguo que está experimentando diversas transformaciones y que se está alimentando recientemente de la irrupción de los medios sociales y de la intensa cobertura televisiva de la migración en la frontera estadounidense. El presente estudio se centra 
en los jóvenes de la comunidad considerando que son los más atentos y expuestos a la información de estas fuentes, y que por su pronta inserción a un campo laboral incierto y su natural proceso de definición de identidad y proyecto de vida, son un grupo social particularmente sensible respecto de la cultura de la migración. Lo que se propone es un primer acercamiento entre la cultura de la migración y la comunicación mediante el elemento conceptual de los imaginarios, con el propósito de demostrar los aportes que tal disciplina es capaz de hacer a una problemática de rotunda contemporaneidad y relevancia.

\section{COMUNICACIÓN, CULTURA E IMAGINARIOS MIGRANTES}

Las relaciones entre la comunicación y la migración han sido ampliamente estudiadas en los destinos migratorios respecto del papel que los medios juegan en los procesos de integración de las poblaciones migrantes, la desterritorialización de sus culturas (García Canclini, 2009) y el mantenimiento de su identidad cultural y vinculación con los territorios de origen (Cornejo, Bellón \& Sánchez, 2008; King \& Wood, 2001; Meyer, 2005). No obstante, el papel de la comunicación en las comunidades expulsoras de migrantes ha sido menos indagado, a pesar de que existen al menos tres relaciones salientes: en primer lugar, una influencia de los medios y la comunicación interpersonal en la creación de una "disposición psicológica" que favorece los movimientos poblacionales, la cual se remonta a las teorías acerca de un efecto de homologación cultural entre los lugares de origen y destino que propicia -sobre todo en las capas medias- una migración de tipo ideológico (Díaz Nosty, 2006; Durand \& Massey, 2003) y anteriormente al trabajo pionero de Daniel Lerner acerca de las transformaciones culturales de Oriente Medio en los años cincuenta y el paso de una sociedad tradicional a una moderna más proclive a la migración (Thompson, 1998). En segundo lugar, la comunicación puede concebirse como un proceso que produce representaciones e imaginarios acerca de los destinos migratorios y los lugares de origen, teniendo como vectores los medios de comunicación, la comunicación interpersonal y los bienes culturales; la información que circula es de naturaleza simbólica por lo que produce apropiaciones que contribuyen en mayor o menor medida a perfilar 
opciones de migración (Hawkins, Minjares, Harris \& Rodríguez De la Gala, 2010). En tercer lugar la comunicación vehicula información de naturaleza instrumental -cómo, cuándo, dónde y con quién cruzar la frontera y asentarse en el lugar de destino- en tanto que funciona como un recurso que facilita los procesos estratégicos involucrados en migrar, ya sea bajo la gestión interesada de agentes -“coyotes", oficiales corruptos- en su calidad de mercancía o de forma solidaria entre migrantes que de esta manera dinamizan y consolidan redes sociales migratorias (Pedone, 2002). Se concibe pues como una información "para migrar" en oposición a la información "acerca de la migración" expuesta previamente (Hawkins et al., 2010).

La exposición aquí se centra más bien en la dimensión simbólica de la comunicación que permite construir imaginarios y representaciones tanto del lugar de destino -sus potenciales ventajas, sobre todo- del proceso migratorio mismo, así como de manera fundamental acerca del entorno propio -en un nivel local o nacional- que permite establecer una diferencia clara y en ocasiones desventajosa entre el "aquí" y el "allá". En ese sentido las comunidades de expulsión migratoria pueden ser concebidas como sistemas desorganizados de circulación de información para y acerca de la migración (Pedone, 2002). La hipótesis central es que las narrativas que diversos agentes introducen a la comunidad a través de múltiples canales de comunicación - charlas, cartas, llamadas o mensajes electrónicos incorporados en envíos y otros bienes materiales- se integran a las narrativas de los medios de comunicación -periódicos locales, programas de televisión- y de otros agentes institucionales -coyotes, autoridades- para crear imaginarios y representaciones acerca de migrar, lo cual tiene consecuencias pragmáticas al fungir como elementos involuntariamente persuasivos hacia dicha decisión e interactuar con otros aspectos propios de la potencialidad migratoria-económicos, afectivos, sociales- que pudieran desencadenar una decisión de movilidad. Esto quiere decir que si bien la información, el conocimiento o los imaginarios mismos no disparan un proceso migratorio per se, como sí lo hace la precariedad económica y/o la existencia de una red migratoria, la presencia de aquellos en tanto recursos que disminuyen la incertidumbre incrementan la probabilidad de migrar para aquellos sujetos que presentan indicios potenciales de hacerlo (Appadurai, 1990). 
Los imaginarios por su parte pueden ser entendidos como construcciones culturales, locales o amplias, conformadas por aspectos racionales e irracionales, objetivos y subjetivos, ideales y reales que están ligadas a la acción (Goycoechea \& Ramírez Gallegos, 2002). Los sujetos se proyectan a sí mismos en los relatos o son "transportados" por ellos en su mundo. Las "imaginaciones" son "narrativas elaboradas a partir de percepciones concurrentes acerca de un evento/estímulo próximo o distante, de recuerdos, historias culturales, leyendas, mitos y experiencias" (Sarbin, citado en Sladkova, 2007, p. 11). A través de la construcción de sus mundos, los seres humanos son capaces de ubicarse a sí mismos no solo con referencia a objetos y eventos que están presentes en su cotidianeidad, sino también en referencia a objetos, lugares y eventos ausentes (Sladkova, 2007).

La importancia de los imaginarios es mayúscula en el contexto de las identidades juveniles modernas en cuanto a su formación reflexiva a partir de la información y el conocimiento que circula en el entorno y a las atribuciones individualistas de los proyectos de vida que conforman. De acuerdo a los teóricos de la identidad, los individuos en modernidad conciben sus vidas como un proyecto biográfico coherente sostenido de manera refleja a lo largo de toda una trayectoria de vida que incorpora los flujos de información y conocimiento como materia prima de posibles modos de vida (vidas imaginadas) y que permite la construcción de una imagen de sí y de los demás (Appadurai, 2001; Giddens, 1995). Así, la información interpersonal y la mediática "pueden proveer a los individuos de medios para explorar formas alternativas de vida de modo simbólico o imaginario ... permitiéndoles de ese modo reflexionar críticamente sobre ellos mismos y sobre las actuales circunstancias de sus vidas" (Thompson, 1998, p. 276). Appadurai (2001) concibe a este rasgo de la modernidad como un "trabajo de imaginación" que piensa está al centro de los proyectos y la acción colectiva (p. 10).

La segunda característica de las identidades contemporáneas es que los individuos tienen que tomar decisiones en un contexto de incertidumbre y turbulencia, en lugar de orden y estabilidad, por lo que los jóvenes se ven constreñidos a "aceptar responsabilidades personales y desarrollar estrategias individuales para manejar el riesgo, en lugar de confiar en el apoyo de las instituciones sociales" (Easthope \& Gabriel, 
2008, p. 174). Los individuos atribuyen sus condiciones y circunstancias actuales a sus propias decisiones y fincan su proyecto de vida en sus propios recursos individuales, en lugar de confiar en el entorno social (Beck, citado en Reguillo, 2009). De esta manera la información y conocimientos consumidos son recursos que conforman los imaginarios con los cuales los migrantes potenciales construyen sus identidades, trayectorias y estrategias de vida; con ello se puede inferir que si la información, conocimiento e imaginarios están orientados a la migración, es probable que su identidad y trayectoria de vida contemplen esta decisión, incluso de manera provisional.

Estos fenómenos son particularmente manifiestos en los jóvenes: recordemos que en las sociedades modernas tales sujetos experimentan una "crisis de identidad", la cual resuelven integrando su pasado infantil $\mathrm{y}$ expectativas futuras en el transcurso de una moratoria psicosocial sin asignación de rol, con la ayuda de agencias socializadoras, entre las que se encuentra los pares, la familia y los medios (Dubet, 1989; Revilla, 2001). La fragilidad de una identidad precaria y en construcción implica una mayor atención y asimilación de los discursos de dichas agencias de cara a una trayectoria futura, incluso en juventudes rurales -como la estudiada aquí- que se asemejan cada vez más a las urbanas en el contexto de una "nueva ruralidad" (Baños, 2003; Durston, 1998).

En su conjunto los aspectos mencionados se vuelven decisivos en la conformación o consolidación en una comunidad de una cultura de la migración, "un sistema integrado de normas, actitudes, creencias, valores y sanciones que regulan la actividad migratoria e informan la decisión de mirar" (Hawkins et al., 2010, p. 163) y que permite a los nuevos migrantes integrarse al flujo con facilidad y manejarse en sus circuitos transnacionales (Durand, citado en Gaspar Bojórquez, 2006) ya sea a través de los mecanismos de socialización familiar -transmisión intergeneracional de capital cultural dentro de las familias -, o en el discurso cotidiano de la comunidad, los imaginarios y representaciones de la migración que circulan en forma de relato oral van introduciendo en la comunidad un sistema de valores y expectativas que naturalizan la decisión de migrar. Todo ello implica que los imaginarios acerca de la migración son elementos fundamentales para la conformación de una cultura de la migración, no solo en lo que corresponde a las actitudes y 
creencias acerca del tránsito y destino - objetos imaginados con base en la información introducida a la comunidad-sino también en lo tocante a los recursos simbólicos que los migrantes potenciales utilizan para construir su identidad y trazar sus trayectorias de vida.

En este sentido los canales interpersonales, los bienes simbólicos y los medios de comunicación funcionan como vectores de estos imaginarios y representaciones. La comunicación interpersonal jugaría tal vez el papel más relevante. Por un lado los migrantes que regresan a la comunidad o que llegan de visita, así como las personas inmersas de alguna manera en una dinámica migratoria - con parientes o amigos que han migrado- les transmiten a los migrantes potenciales narrativas acerca de su experiencia o conocimiento del fenómeno. Por otro lado ciertos agentes interesados en diseminar información en la comunidad, tales como los llamados coyotes o agencias de turistas que participan en el tráfico ilegal de personas, contribuyen a la construcción de imaginarios con finalidades estratégicas de persuasión y ganancia.

Los bienes simbólicos y la cultura material en general serían también fuente de representaciones acerca de migrar. La renovada arquitectura local, elaborada con las remesas de los migrantes, condensa para "los que se quedan" un significado de prosperidad y movilidad social como recompensa de haber migrado, además de un posible efecto de privación relativa (Durand \& Massey, 2003). En virtud de su capacidad para generar apropiaciones subjetivas que conducen a inclusiones y/o distinciones culturales y grupales (García Canclini, 1993, 2009; Santillán \& Ramírez, 2004), los bienes simbólicos que los emigrantes o los parientes de migrantes ostentan en la comunidad -en forma de vestimenta, aparatos electrónicos o contenidos de medios- revisten un valor/ signo de estatus (Baudrillard, 2007) que pudiera estimular significaciones acerca de un cambio en el estilo de vida de sus poseedores, asociado a la prosperidad y la "vida moderna".

Como dispositivos de conformación de una cultura (Rodríguez Salazar, 2009) que reflejan, crean y transforman representaciones sociales (Farr, 1998), los medios de comunicación ayudan -desde la ficción y la información - a construir dichos imaginarios, puesto que le permiten a los migrantes potenciales "una visión previa de los destinos con precisiones inimaginables en el pasado" (Díaz Nosty, 2006, p. 10) aunque 
ello implique en ocasiones una perspectiva distorsionada de los mismos. En la lógica de las "medias vistas" de Appadurai (1990), crean también un efecto de contraste: los medios difunden "imágenes de bienestar que no pueden ser atendidas por el estándar de vida nacional" (Díaz Nosty, 2006 , p. 5) a las que se unen otros valores de modernidad que contrastan con las carencias locales y con una "visión del espacio local como un mundo en crisis y clausurado para la realización de proyectos de vida" (Herrera, 2002, p. 90). Al mismo tiempo los medios diseminan información que conforma imaginarios negativos acerca del lugar de residencia -a escala local, regional o nacional- con relación a problemáticas como la pobreza, la inseguridad o la corrupción, lo que pudiera dar pie a negar o menospreciar las expectativas locales de futuro.

El impacto de estos aspectos es mayor cuando las comunidades de origen y destino se compenetran en la formación de una comunidad transnacional que se comunica de manera permanente a través de tecnologías de comunicación e intercambio de mercancías (Brettel \& Hollifield, 2008; Schimitter, 2008). Así, la combinación de mensajes que provienen de estos canales, aunque en ocasiones superpuestos o contradictorios, se convierten en recursos clave en la decisión de migrar:

Los individuos, familias y comunidades enteras aceptan algunas, rechazan otras y las combinan en los "mundos imaginados" lejanos de su realidad situada ... Si este mundo imaginado se convierte en poderoso y persuasivo, algunos individuos pudieran proyectarse a sí mismos en el rol de un migrante en un destino deseado, y esta imaginación pudiera llegar a la migración concreta. Otros pudieran imaginarse a sí mismos en el difícil recorrido hacia los EE.UU. y pudieran elegir quedarse en su comunidad (Sladkova, 2007, p. 191).

Estudios empíricos de diversas regiones migratorias constatan la existencia de imaginarios en las comunidades de expulsión migratoria, cuyas piezas provienen de diversas fuentes: testimonios de los padres, medios de comunicación, entorno escolar y comunitario y "sus propias ideas juveniles del mundo" (Carrillo, 2005, p. 372), mismas que compiten por la atención de la gente (Sladkova, 2007). Los jóvenes de las comunidades estudiadas manifiestan visiones utópicas de las oportunidades y ventajas que pueden encontrar en otros países. Describen los luga- 
res de destino basados en imaginarios del capitalismo global, dentro de una jerarquía de progreso y modernidad en donde abundan discursos de "avanzado" y "atrasado", elaborando con ello versiones de una "buena vida" (Mar, 2005). Hay narrativas positivas transmitidas a través de cartas, llamadas telefónicas, videos, fotos y conversaciones por parte de los migrantes, incluidos los propios padres, acerca de la consecución rápida de un empleo de alta remuneración, de muchos más atractivos para ocupar el tiempo libre y una mayor libertad que en la comunidad (Carrillo, 2005; Echeverri, 2005; Goycoechea \& Ramírez Gallegos, 2002; Hawkins et al., 2010; Patiño, 2005). De manera complementaria los jóvenes reciben mensajes de los medios sobre la modernidad, individualidad, libertad y bienes asociados con una "apropiada" juventud (Beazley, 2007).

De manera complementaria se presentan en las comunidades de origen narrativas acerca de la facilidad de cruzar la frontera en virtud de la corrupción de los oficiales fronterizos. Se supone que tales narrativas son creadas y distribuidas por los coyotes locales -o en su caso, las personas que están relacionadas con el negocio de la movilidad de personas- para competir por clientes en la comunidad (Sladkova, 2007).

Desde el punto de vista de la cultura material, los jóvenes atestiguan que sus padres, tíos, tías y vecinos regresan a casa con suficiente dinero para comprar bienes de consumo y construir casas de materiales duros, que son signos de prosperidad y riqueza, por lo que pudieran constituir un efecto de demostración (Beazley, 2007; Goycoechea \& Ramírez Gallegos, 2002; Hawkins et al., 2010; Vega, 2006). Asimismo, la influencia de los migrantes que regresan para las fiestas o vacaciones es significativa para los jóvenes de las comunidades. "El regreso de migrantes desplegando nuevos vestidos y bienes materiales provee a los otros jóvenes con información sobre culturas juveniles globales, y actúan hasta cierto punto como una ventana al mundo moderno" (Bey, citada en Punch, 2007, p. 101). Esta función es particularmente importante en contextos rurales en donde el acceso a los medios y estilos de vida externos son muy limitados, y las mercancías y objetos que se consumen expresan en mayor medida la sensación de movilidad social (Zilberg, 2003).

No obstante los migrantes de retorno y los medios de comunicación también socializan narrativas negativas acerca de las condiciones de 
explotación, trabajo duro, privación de libertad, falta de tiempo libre y racismo en el lugar de destino (Beazley, 2007; Carrillo, 2005; Hawkins et al., 2010; Sladkova, 2007; Vega, 2006), además de otros aspectos como el tráfico ilegal o "coyoterismo", la desintegración familiar, o la vida a la sombra del estatus migratorio ilegal (Carrillo, 2005). Asimismo circulan narrativas acerca de los lugares de destino como sitios que "corrompen" los valores morales de las personas, de mayor libertinaje y desorden sexual (Carrillo, 2005).

Respecto de las consecuencias pragmáticas de estos imaginarios, encontramos poca evidencia que permita establecer en qué medida contribuyen a una decisión migratoria. El estudio de Díaz Nosty (2006) encontró que de 600 inmigrantes de seis países entrevistados en España en 2006, $15.2 \%$ declaró que lo que veían en televisión o escuchaban en radio en sus países de origen fue de "mucha influencia" para migrar, junto con 13\% que declaró lo mismo para el caso de periódicos, revistas y otros medios impresos. Estos porcentajes se elevan a $26.8 \%$ y $16.4 \%$, respectivamente, para los migrantes sin redes en el lugar de destino, de manera que la principal fuente de información fueron los testimonios de familiares y amigos que habían residido o residían en ese momento en dicho país; estas cifras desde luego no son concluyentes, particularmente porque son los mismos entrevistados los que declaran haber sido influidos. No obstante sí disponemos de datos cualitativos que permiten apoyar la hipótesis del contraste subjetivo entre las condiciones externas imaginadas y las locales. Hay una especie de consenso juvenil de que no es posible "salir adelante" en su comunidad de origen y que en cambio es posible escapar de la pobreza e incrementar el estatus económico y social yéndose al extranjero (Beazley, 2007; Carpena-Méndez, 2007; Echeverri, 2005; Hawkins et al., 2010; Punch, 2007). "El marco local de oportunidades no abastece las proyecciones de realización personal en el tiempo" (Goycoechea \& Ramírez Gallegos, 2002, p. 37). En el nivel macro, por ejemplo, solo $10.3 \%$ de jóvenes mexicanos les gusta su país por las oportunidades de vida que presenta, en contraste con $57.8 \%$ que lo valoran por su cultura y tradiciones (León, 2008).

Tales datos sugieren que los imaginarios que dimos cuenta páginas atrás contribuyen a establecer o reafirmar determinadas creencias y actitudes hacia la migración, lo cual pone en evidencia la manera en 
que favorecen una "cultura de la migración" en los lugares en los que se manifiestan. En ese sentido aunque el objeto mismo de la identidad no fue explorado en los hallazgos compendiados, es posible afirmar que muchos de los elementos descritos en efecto se incorporan a las definiciones de identidad y están contemplados en los imaginarios de futuro de los jóvenes; particularmente ante su inminente inserción a un mercado laboral incierto, su proceso de definición identitaria, en el periodo juvenil (Bushin, Ansell, Adriansen, Lahteenmaa, \& Panelli, 2007; Urteaga, 2008), y su propio deseo de autosuficiencia.

\section{MÉTODO}

La aproximación al tema de estudio es de tipo cualitativa, puesto que el acento está puesto en la exploración del sistema de creencias, significados e imaginarios sociales que entran en juego en la vivencia de la migración desde el contexto social y el punto de vista de los actores involucrados.

Tunkás, la comunidad de estudio, está localizada a 70 kilómetros de Mérida, la capital de Yucatán. Aproximadamente 11\% de sus 2 mil 828 habitantes radican en Estados Unidos. Se trata de una comunidad predominantemente rural, puesto que $51 \%$ de las personas en edad productiva se dedican a la agricultura (Gell-Redman, Andrade, Martell \& Jiménez Pacheco, 2010) y la mayoría de sus habitantes viven en condiciones de pobreza: el promedio de escolaridad es de 5.7 años y $84.6 \%$ de la población vive hasta con dos salarios mínimos (CONAPO, 2006); $56 \%$ de las viviendas presentan algún tipo de hacinamiento (INEGI, 2010). Esto implica una corriente migratoria de origen económico, aunque también se ha desarrollado una red migratoria entre dicha comunidad y la ciudad de Anaheim, California que mantiene relativamente vivos los flujos migratorios incluso en tiempos de crisis económica o fuertes restricciones al cruce de la frontera (Estrella, 2010).

Para integrar la muestra de jóvenes se consideraron los criterios empíricos de investigaciones previas en la comunidad (Hawkins et al., 2010), así como criterios teóricos acerca de los migrantes potenciales (Chiswick, 2008; Herrera, 2006; Pacione, 2009). El rango de edad en que se consideró a un entrevistado como joven comprende entre los 14 
y los 28 años de edad (esta última es la edad promedio de matrimonio en la comunidad de estudio, lo cual cambia la potencialidad migratoria). Utilizamos dos cohortes para integrar la muestra: el primero se definió entre los 14 y los 18 años de edad, y el segundo entre los 19 y 28. Los 18 años es una edad significativa para separar los cohortes porque es la edad promedio de la primera migración interna -hacia otros puntos del país- y el umbral en donde hay más tunkaseños laborando que estudiando. Otros criterios de inclusión fueron que sean varones ${ }^{3}$ y que al momento de la entrevista al menos un familiar radicara en Estados Unidos o haya tenido experiencia migratoria, que hubiesen terminado la secundaria y que no tengan planes de matrimonio; atributos que en su conjunto los caracteriza como migrantes potenciales, inmersos en una socialización familiar y en un proceso de definición de proyecto de vida. Bajo un criterio de saturación teórica -el momento de la investigación en el que ya no se obtiene información nueva- fueron entrevistados en profundidad 12 jóvenes para cada uno de los grupos.

En congruencia con lo expuesto en el apartado teórico, presentamos los datos empíricos organizados sobre cuatro ejes de imaginarios y representaciones: acerca de la migración misma, del lugar de origen, del lugar de destino, del tránsito por la frontera y aquellas incorporadas a los bienes simbólicos que envían los migrantes. Adicionalmente indagamos en las trayectorias de vida de los entrevistados para observar en qué medida los imaginarios se intersectan en los proyectos de vida de los migrantes, independientemente de si hay una decisión migratoria de por medio. En general queremos establecer en qué medida los imaginarios y representaciones fungen como recursos en la construcción de una cultura de la migración, una serie de creencias y actitudes colectivas proclives a desencadenar una decisión migratoria.

3 Excluimos intencionalmente a las mujeres porque representan en la comunidad una fracción mínima de sujetos con disposición a migrar, además de que lo hacen más bien por razones familiares, alejadas de la problemática central que aquí se expone; en otras palabras, la inclusión de las mujeres ameritaría un aparato teórico específico. 


\section{HALLAZGOS}

Los datos de los dos grupos serán expuestos de manera indistinta puesto que los patrones que emergieron son similares, salvo cuando haya que precisar alguna particularidad en alguno de los dos grupos.

\section{Acerca de la migración}

Los jóvenes perciben consecuencias tanto positivas como negativas de migrar. Los entrevistados conocen de manera directa o vicaria las consecuencias de migrar desde el punto de vista del impacto emocional; los hijos de migrantes señalan la ausencia de sus padres como una afectación emocional o una pérdida, particularmente en los momentos emblemáticos de la vida familiar -como los cumpleaños o la celebración de los "XV años"- así como el riesgo de desintegración familiar.

Las imaginaciones acerca de las consecuencias positivas de migrar son más variadas y están relacionadas con la retroalimentación que parientes, pares y conocidos les dan a los jóvenes. La mayoría tiene que ver con la posibilidad de incrementar el patrimonio en la misma comunidad, ya sea hacerse de una casa espaciosa, de propiedades, ranchos o ganado. Abundan casos exitosos de vecinos, familiares o conocidos que consiguen propiedades y a veces regresan después de conseguirlas, algo que es observado directamente por los jóvenes, o bien son informados de ello, lo que les da la impresión de que se van precisamente para eso. Un joven incluso deduce que la consecución de una vivienda para el migrante es signo de que la decisión de migrar fue la adecuada. La elevación del ingreso y la calidad de vida en Estados Unidos, aspectos equiparados por cierto, es otra consecuencia positiva mencionada con cierta frecuencia. Solo un entrevistado mencionó como consecuencia positiva de migrar la posibilidad de ayudar a sus familiares.

En el grupo de 14 a 18 años las posiciones están polarizadas: la mitad del grupo apoya la idea de migrar para ganar más dinero u obtener bienes de consumo, y la otra mitad reprueba la idea por las razones negativas mencionadas o por el riesgo implicado. No obstante estas posiciones, el tema de la migración en general parece de una importancia relativa en estos jóvenes estudiada. La mitad de los entrevistados 
no están muy atentos al tema -no saben cómo cruzar la frontera, por ejemplo- no hablan de él con sus pares, familiares o consumen noticias referentes al mismo.

\section{Acerca del cruce de la frontera}

Casi todo el grupo concibe al acto del cruce de la frontera como riesgoso, siendo el peligro de morir el más grave. Cerca de la mitad del grupo puede describir formas específicas de morir en la frontera, como la deshidratación, los ataques de animales desérticos, el abandono por parte de "la migra" en el desierto o los asesinatos perpetrados por agentes migratorios, así como otros fenómenos tales como los robos con violencia, la vigilancia reforzada de la patrulla fronteriza - que hace más difícil el cruce- o el perderse en el desierto. En ocasiones hay relatos muy precisos acerca de la violencia en la frontera e incluso uno de los entrevistados se ha retractado de migrar a causa de dicha percepción:

Ahorita hay un grupo de gente que si estás entrando, a veces nomás de malos te matan en la frontera (Mario, 17 años).

La mayoría de la información relatada acerca de acontecimientos recientes en la frontera y en Estados Unidos proviene de los medios de comunicación, y en segunda instancia de los relatos que circulan en la comunidad o por parte de familiares (padres, primos, hermanos) con experiencia migratoria. La mitad del grupo observó noticias de televisión acerca del reforzamiento de las condiciones restrictivas de la frontera (muros, vigilancia), lo que atañe a la llamada "Ley Arizona" y de modo ampliamente destacado y consensuado, los asesinatos que se presentan en la frontera y que tuvieron cobertura noticiosa en días previos a las entrevistas.

Aunque varios entrevistados pudieron describir con detalle "cómo se le hace" para llegar a la frontera y qué recursos son necesarios para migrar - lo que denota un alto grado de información instrumental- dicha opción se desestima ante las narrativas previamente descritas. Casi la mitad del grupo opina que el intentar cruzar la frontera es un esfuerzo muy arriesgado que hace perder dinero a las personas -mientras que este escasea en la comunidad-suscitando reflexiones como la siguiente: 
Yo preferiría quedarme aquí a arriesgar mi vida. Con poco, pero sé que estoy vivo, con mi familia. Es mejor estar acá (Julio, 22 años).

\section{Acerca del lugar de destino (Estados Unidos)}

Los entrevistados dibujan un imaginario positivo acerca de Estados Unidos. La mitad del grupo afirma que las condiciones de empleo son favorables en el país del norte, particularmente respecto de los salarios de los que se puede vivir de manera satisfactoria y acumular dinero; esta es la condición básica por la que los migrantes están ahí, según varios entrevistados. Se argumenta un valor del dólar más alto que el peso y un esquema de pago por hora, y no por jornada como en México, aspectos que ellos suponen que benefician al trabajador. Existen incluso relatos muy atractivos de salarios prácticamente irreales, probablemente difundidos con fines de prestigio por parte de los migrantes:

Un amigo que se fue ... me dijo que en eso de la construcción debería estar ganando 25 dólares la hora (Josué, 15 años).

La mitad del grupo comentó que los migrantes "viven bien ahí" e incluso hay ciertos entrevistados que han escuchado que "viven mejor" que en Tunkás. Especialmente las condiciones de habitación son mejores, porque las casas son más espaciosas, "bonitas" y equipadas. El grupo de 14 a 18 años destaca con mayor énfasis los bienes de consumo que pueden adquirirse en Estados Unidos -computadoras, videojuegos, tecnología e incluso armas- o las condiciones de "modernidad" y "progreso" que ahí tienen -hoteles, aviones, casas lujosas, etc.-. Estos jóvenes afirman que además de los comentarios de los migrantes que regresan, o las fotografías que les envían parientes y amigos mediante Internet, han visto estos aspectos en películas estadounidenses.

A la par, los entrevistados también sostienen imaginarios y representaciones negativas acerca de las condiciones de vida de los migrantes en Estados Unidos, particularmente acerca de la supuesta falta de libertad que los migrantes tienen en ese país a partir de su condición migratoria ilegal. La narrativa es que los migrantes solo van de su trabajo a su casa, y ahí se "encierran" para no ser deportados por las autoridades migratorias. No "salen" -no aprovechan la oferta cultural o de esparcimiento 
del lugar-y por lo tanto no disfrutan de su libertad ("no puedes vivir la vida"). Aunado a la amenaza de las autoridades está el riesgo de ser agredidos, asaltados o asesinados por bandas delictivas, de manera que la mencionada falta de libertad viene acompañada de sensaciones de temor, fragilidad y riesgo continuo por parte de los migrantes. Sobresalen también las dificultades de adaptación -a alimentarse, por ejemplo-, la dureza del trabajo y el dolor en general de vivir ahí ("ahí se sufre"), precariedad por falta de empleo o una relación desfavorecedora entre ingreso y gastos. Unos entrevistados comentan que las condiciones de empleo son precarias: los empleadores no toleran inasistencias y despiden a un empleado a su primera falta, y los sueldos son relativamente bajos. Otro aspecto enfatizado por el grupo es el de la condición de discriminación racial que viven los migrantes, que deriva en maltrato por parte de autoridades y empleadores, rechazo del idioma español, y aspectos de violencia. También existen argumentos distorsionados que confunden aspectos de discriminación con esquemas laborales restrictivos, que no necesariamente implican desigualdad de trato. Estas narrativas hacen concluir, cuando menos a un par de entrevistados, que la vida en Tunkás es mejor que en Estados Unidos.

\section{Acerca del lugar de origen}

En contraste con las ciudades -en especial para aquellos entrevistados que conocen Mérida, capital de Yucatán o Cancún y Playa de Carmen, en Quintana Roo- Tunkás se concibe como un lugar tranquilo y seguro para la mayoría de los sujetos, exento de los riesgos urbanos tales como el tráfico, la drogadicción, el vandalismo, los pleitos callejeros, los insultos en las calles, las bandas, los asaltos o los asesinatos; aspectos que amenazan la seguridad de las personas y que definen a la experiencia urbana como peligrosa. En la comunidad en cambio es posible utilizar libremente su tiempo de esparcimiento sin temor a ser vulnerado. Algunos entrevistados manifestaron sentirse muy bien en la comunidad, con una sensación incluso de privilegio.

No obstante existen también algunos aspectos negativos que los jóvenes destacan acerca de la comunidad, particularmente su precariedad económica. La mayoría de los entrevistados resiente la falta de empleo y de remuneración satisfactoria. Destacan que lo que puede obtenerse 
en el campo es muy poco, además que por esa vía es muy difícil "moverse" económicamente:

No se puede "prosperar", se gana muy poco para comprar un terreno y una casa (Mateo, 20 años).

Un entrevistado considera que esa es la principal razón por la que no se quedaría a vivir ahí.

\section{Bienes simbólicos}

En cuanto al consumo de bienes simbólicos, la vestimenta es el aspecto más enfatizado. Una tercera parte de los entrevistados afirman que ha vestido en algún momento tipo "cholo" (pantalón y camisa anchas, gorra y aretes) cuando eran adolescentes, pero dejaron de hacerlo al crecer, en ocasiones a partir de que la gente empezaba a hablar de manera despectiva sobre ello. Varios entrevistados admiten que empezaron a vestir así, imitando a sus hermanos mayores, grupos de referencia, figuras de la televisión o bien de los migrantes -entre ellos parientes o primos- que venían de Estados Unidos y/o que enviaban ropa de ese estilo; los migrantes les decían que era la moda y los entrevistados seguían esa influencia. Un par de entrevistados mencionaron que aún visten así, pero solo en ocasiones especiales como fiestas o fuera de su comunidad, como cuando van de visita a las ciudades de Mérida o Playa del Carmen. Cuando hay eventos religiosos u oficiales entonces cambian a una ropa que denominan como "normal". Ciertos entrevistados interpretan de manera reflexiva el dejar de vestir tipo cholo como una fase de maduración antes de integrarse a la sociedad.

En el grupo de 14 a 18 años el valor de los bienes simbólicos está más acentuado. El vestir de determinada manera o usar determinados objetos significa en la mitad de los entrevistados una sensación de aceptación, prestigio o distinción al señalar explícitamente que no muchos los tienen, o bien que son muy costosos. Afirman que sus amigos o personas del sexo opuesto les dicen que la vestimenta o los zapatos "se ven bien", "están bonitos" o "les gustaría tenerlos". Casi la mitad del grupo menciona que su vestimenta proviene de los envíos de sus padres, hermanos o tíos, aunque en ocasiones deciden utilizarlos poco 
o no utilizarlos en absoluto porque no les agrada. Los bienes de consumo provenientes de Estados Unidos o de otros lados son relativamente importantes para los entrevistados, a tal grado de que si los dejaran de recibir, los intentarían buscar por su cuenta en Cancún o en Mérida.

Por otro lado, la ostentación de bienes de consumo de parte de los migrantes que regresan de Cancún o Estados Unidos -computadoras, teléfonos celulares, juegos de video, vestimenta, etc.- es rechazada por varios de nuestros entrevistados. Solo en el grupo de 14 a 18 años, algunos manifiestan que estos les llaman la atención e incluso uno de ellos aspira a tenerlos.

\section{Trayectoria de vida, aspiraciones y estrategias}

Casi todos los jóvenes entrevistados mencionaron la aspiración de ser profesionistas, sin un patrón predominante en cuanto al tipo de profesión: maestro, psicólogo, administrador, cocinero -esto influido por la información de que es una ocupación cotizada en Cancún- o bien ingeniero en computación, en el sector de la construcción o en seguridad pública en el grupo de 18 a 25 años. Esta definición es menos clara en el grupo de 14 a 18, puesto que una tercera parte definió una profesión específica, y el resto esbozó más bien una ocupación u oficio. Varios entrevistados de ambos grupos expresaron su rechazo a trabajar en el campo, puesto que no les gusta la dureza física de la labor.

Las aspiraciones de los jóvenes son de tipo material y personal. Estas últimas fueron las menos mencionadas, ya que solo la mitad del grupo expresó deseos de formar una familia y tener un par de hijos como máximo. Las aspiraciones materiales en cambio son centrales. La principal de ellas y que básicamente es consensuada por todo el grupo es la adquisición de una vivienda en Tunkás ya sea "normal" -respecto del estándar de vivienda de la comunidad -o extraordinaria -que supera ese estándar con ciertas comodidades-. Una tercera parte del grupo quisiera tener su casa en Mérida, Playa del Carmen u otra ciudad de la República - pensando en que migrarían para buscar empleo- y al resto le gustaría construir su casa en Tunkás. Casi todos los entrevistados se ven trabajando, unos en una carrera ascendente y la mayoría con un buen salario que les permita no privarse de los bienes básicos. 
Para lograr esto las personas plantean diversas estrategias (que son menos claras en el caso del grupo de 14 a 18 años). La principal es la búsqueda de un empleo bien remunerado, lo que implica migrar a Estados Unidos, donde algunos entrevistados tienen información que los salarios son altos. Otros piensan migrar a Cancún, Playa del Carmen o Mérida, reunir el dinero y enviarlo para construir su casa; pretenden regresar a Tunkás ocasionalmente a visitar a sus parientes. Los entrevistados migrarían a través de los recursos que tienen en otros lados -familiares que les dan alojamiento, o amigos que les pueden buscar empleo-. En el caso del grupo de 14 a 18 años, algunos entrevistados afirman que trabajarían en el corto plazo fuera de Tunkás y regresarían después de unos tres años de trabajo. En general hay un discurso de esfuerzo personal que se traduciría en el logro de sus metas -a mayor empeño, mayores logros-:

Dar lo mejor de mí en la escuela, tener un empleo y esforzarme un poco más cada día (Pablo, 15 años).

\section{DISCUSIÓN Y CONCLUSIONES}

En lo general los datos revelan en primera instancia la confluencia de narrativas positivas y negativas en un imaginario complejo acerca de la migración. Los tres elementos que destacamos como objetos imaginados, el acto de migrar, el destino y el origen, no se orientan en un solo sentido. En primera instancia las representaciones negativas sobre el acto de migrar, específicamente en el ámbito emocional, se compensan con representaciones positivas tales como la posibilidad de incrementar el patrimonio - un aspecto por cierto relacionado con la cultura material- así como la elevación del ingreso y la calidad de vida. Como en otras investigaciones reseñadas, se piensa que migrar pudiera traer condiciones de empleo, remuneración y de vida en general óptimas. Cruzar la frontera en cambio se imagina como un proceso más sombrío, cargado de riesgo y peligro. De la misma manera el imaginario negativo acerca de vivir en Estados Unidos que encontramos en ciertas investigaciones empíricas previas se confirma también en Tunkás: privación de libertad, trabajo arduo, 
riesgo y amenaza continua por parte autoridades, delincuentes, empleadores y ciudadanos racistas.

En contraste, la representación del lugar de origen es un elemento importante en las negociaciones subjetivas acerca de migrar. Hay un pronunciado sentido de arraigo y una concepción del sitio como apacible, protector y seguro, que contrasta con las representaciones acerca de las ciudades como lugares hostiles. Sin embargo, como en los trabajos empíricos previamente comentados, Tunkás se concibe como un lugar prácticamente clausurado de oportunidades económicas de empleo o remuneración adecuados.

La comunicación interpersonal y la masiva son centrales en la conformación de estos imaginarios. Las narrativas positivas y negativas acerca del acto de migrar y de Estados Unidos provienen fundamentalmente de migrantes que han tenido experiencias del tránsito y destino migratorio. Aunado a ello, la información noticiosa negativa es fundamental en las imaginaciones de los jóvenes acerca de un tránsito fronterizo y condiciones de vida adversas en el país del norte, aunque es necesario tomar en cuenta que al momento de la investigación se suscitó una tematización noticiosa al respecto, que como tal es pasajera y de una influencia tenue sobre la capacidad de persuasión interpersonal (Balle, 1991). No obstante los aspectos mencionados influyen incluso en la representación del lugar de origen, del entorno rural propio: aunque esta pudiera parecer sustentada tan solo en la subjetividad, la valoración positiva de seguridad y la negativa de precariedad surge en contraste con los sitios urbanos que han sido construidos intersubjetivamente con base en narrativas comunicadas.

La cultura material se caracteriza en los datos como un elemento simbólico importante en las representaciones de la migración y sus recompensas. La edificación de viviendas o su remozamiento, así como la adquisición de propiedades, son símbolos de éxito y gratificación para aquellos que acometieron la empresa migratoria. Para los más jóvenes los bienes simbólicos y el atuendo que introducen los migrantes o que son el resultado de trabajar y vivir en el extranjero, representan objetos de distinción, inclusión y prestigio que se aprecian en su capacidad de conferir estatus al portador. De ahí que se vuelvan objetos imitados y buscados por los mismos. En los grupos menos 
jóvenes esta distinción deja de tener efecto, posiblemente porque estos dejan de tener una "moratoria" y son coercionados a integrarse de lleno a las reglas del "mundo adulto" (Brito, 2002).

La presencia de estos elementos, particularmente los negativos, tiene consecuencias pragmáticas en una buena parte de los entrevistados que han tomado la decisión de no aprovechar por el momento las redes migratorias de las que disponen en Estados Unidos en ese sentido aquellos han orientado una parte de la "cultura de la migración" respecto de las creencias y actitudes que manifiestan hacia el hecho de migrar a un destino internacional, desactivando momentáneamente una predisposición. No obstante parecen haber otros elementos culturales también trastocados por los imaginarios: respecto del proyecto de vida de los jóvenes, destaca la búsqueda de autosuficiencia mediante la consecución de una carrera profesional, un empleo bien remunerado y una vivienda propia que les permita desenvolverse fuera de la autoridad de los padres. En esa búsqueda marcadamente individualista no se contemplan opciones relacionadas con la economía local -en el campo-y se contraponen en cambio posibilidades de desarrollo urbanas, propias de los proyectos de vida trazados por identidades modernas, cuyos elementos claramente no se encuentran en esta comunidad predominantemente campesina, y que tuvieron que ser introducidos a la comunidad. Incluso la fijación del grupo de 14 a 18 años en los bienes de consumo que se pueden adquirir en Estados Unidos o la verosimilitud que le otorgan a la ficción cinematográfica respecto de las características reales de los estadounidenses es señal de la participación de los bienes simbólicos en su definición identitaria, así como la permeabilidad de sus creencias sobre lugares remotos a los imaginarios propuestos por los medios.

Es así como la información y el conocimiento que circulan en Tunkás en forma de imaginarios intervienen en la toma de decisión migratoria: de manera directa le han servido a los jóvenes para determinar que el destino migratorio habitual es por ahora inconveniente para acometer la migración, y en ese sentido han reorientado en cierta medida la cultura de migración al modificar las actitudes y creencias hacia aquél. De una manera más indirecta posiblemente los jóvenes se han proyectado en imaginarios de estilos de vida urbanos y modernos, con componentes de adquisitividad, profesionalización e independen- 
cia, configurando proyectos a futuro que necesariamente contemplan la migración interna para cumplirlos, incluso en contra de las sensaciones subjetivas de estabilidad y seguridad que provee el lugar de origen.

Las relaciones entre comunicación y la cultura de la migración en las comunidades de expulsión necesitan profundizarse en varias direcciones, sobre todo la estructura y contenido específico de las narrativas que conforman los imaginarios, las negociaciones que los migrantes potenciales realizan a partir de ellos, o la jerarquía que ocupan las fuentes -en donde sería pertinente una problematización en clave de hegemonía y poder-, sin olvidar desde luego las particularidades de la juventud, especialmente sensible a la movilidad y la incertidumbre. Esperamos ofrecer un primer paso en esa fructífera dirección.

\section{Bibliografía}

Appadurai, A. (1990). Disjuncture and difference in the global cultural economy. Theory, Culture \& Society, 7, 295-310.

Appadurai, A. (2001). La modernidad desbordada. Dimensiones culturales de la globalización. Montevideo, Uruguay: Trilce.

Balle, F. (1991). Comunicación y sociedad, evolución y análisis comparativo de los medios. Bogotá, Colombia: Tercer Mundo Editores.

Baños, O. (2003). Modernidad, imaginario e identidades rurales: el caso de Yucatán. México: El Colegio de México.

Baudrillard, J. (2007). Crítica de la economía política del signo. México: Siglo XXI.

Beazley, H. (2007). The Malaysian orphans of Lombok. Children and young people's livelihood responses to out-migration in eastern Indonesia. En R. Panelli, S. Punch \& E. Robson (Eds.), Global perspectives on rural childhood and youth. Nueva York, EE.UU.: Routledge.

Brettel, C., \& Hollifield, J. (2008). Migration theory. Talking across disciplines. En C. B. Brettel \& J. F. Hollifield (Eds.), Migration theory. Talking across disciplines. Nueva York, EE.UU.: Routledge.

Brito, R. (2002). Identidades juveniles y praxis divergente; acerca de la conceptualización de la juventud. En A. Nateras Domínguez (Ed.), Jóvenes, culturas e identidades urbanas. México: UAM-Iztapalapa/ Miguel Ángel Porrúa. 
Bushin, N., Ansell, N., Adriansen, H. K., Lahteenmaa, J. \& Panelli, R. (2007). Reflecting on contexts and identities for young rural lives. En R. Panelli, S. Punch \& E. Robson (Eds.), Global perspectives on rural childhood and youth. Nueva York, EE.UU.: Routledge.

Carpena-Méndez, F. (2007). "Our lives are like a sock inside out". Children's work and youth identity in neoliberal rural Mexico. En R. Panelli, S. Punch \& E. Robson (Eds.), Global perspectives on rural childhood and youth. Nueva York, EE.UU.: Routledge.

Carrillo, C. (2005). El espejo distante. Construcciones de la migración en los jóvenes hijos e hijas de emigrantes ecuatorianos. En G. Herrera, C. M. Cristina \& A. Torres (Eds.), La migración ecuatoriana: transnacionalismo, redes e identidades. Quito, Ecuador: FLACSO Ecuador.

Chiswick, B. (2008). Are inmigrants favorably self-selected? En C. Brettel \& J. Holliflied (Eds.), Migration theory. Talking across disciplines. Nueva York, EE.UU.: Routledge.

Consejo Nacional de la Población-CONAPO. (2006). Índices de Marginación. Recuperado el 18 de junio de 2011 de www.conapo.gob.mx/ publicaciones/indice2005.htm.

Cornejo, I., Bellón, E. \& Sánchez, E. (2008). En la punta de la lengua: narrativas sobre identidad y migración en diálogo con "La Voz de los Mayas”. En R. Enríquez Rosas (Ed.), Los rostros de la pobreza. El debate (Vol. V). México: UIA.

Díaz Nosty, B. (2006). Los medios de comunicación en la experiencia migratoria latinoamericana. Madrid, España: Fundación Telefónica.

Dubet, F. (1989). De la sociología de identidad a la del sujeto. Estudios Sociológicos, VII (21).

Durand, J., \& Massey, D. (2003). Clandestinos migración México-Estados Unidos en los albores del siglo XXI. México: Miguel Ángel Porrua/UAZ.

Durston, J. (1998). Juventud rural en América Latina: reduciendo la invisibilidad. En J. A. Padilla Herrera (Ed.), La construcción de lo juvenil. México: Causa Joven/CIEJ.

Easthope, H. \& Gabriel, M. (2008). Turbulent lives: Exploring the cultural meaning of regional youth migration. Geographical Research, 46 (2), 172-182. 
Echeverri, M. (2005). Fracturas identitarias: migración e integración social de los jóvenes colombianos en España. Migraciones Internacionales, 3 (1), 141-164.

Estrella, W. (2010). El papel de la migración internacional en los procesos de comunicación interpersonal entre los jóvenes no migrantes de Tunkás, Yucatán. Tesis de licenciatura no publicada, UADY, Mérida, Yucatán, México.

Farr, R. (1998). Las representaciones sociales. En S. Moscovici (Ed.), Psicología social II. Barcelona, España: Paidós.

García Canclini, N. (1993). El consumo cultural y su estudio en México: una propuesta teórica. En N. García Canclini (Ed.), El consumo cultural en México. México: CONACULTA.

García Canclini, N. (2009). Consumidores y ciudadanos. Conflictos multiculturales de la globalización. México: Debolsillo.

Gaspar Bojórquez, A. L. (2006). Rehacer el tejido de Penélope: mujeres y reproducción de la emigración. En M. A. Rebeil (Ed.), XIII Anuario de la Investigación de la Comunicación CONEICC (pp. 402-425). México: Universidad Anáhuac del Norte/UIA León/UIA Ciudad de México.

Gell-Redman, M., Andrade, E., Martell, A. \& Jiménez Pacheco, Z. (2010). Inhabiting two worlds: Tunkaseños in the transnational labor market. En W. Cornelius, D. Fitzgerald, P. Lewin Fischer \& L. Muse-Orlinoff (Eds.), Mexican migration and the US Economic crisis. A transnational perspective. San Diego, CA, EE.UU.: Center for Comparative Inmigration Studies/University of California San Diego.

Giddens, A. (1995). Modernidad e identidad del yo. El yo y la sociedad en la época contemporánea. Barcelona, España: Península.

Goycoechea, A. \& Ramírez Gallegos, F. (2002). Se fue, ¿ ¿ volver? Imaginarios, familia y redes sociales en la migración ecuatoriana a España (1997-2000). Íconos. Revista de Ciencias Sociales, (14), 32-45.

Hawkins, B., Minjares, Y., Harris, L. \& Rodríguez De la Gala, J. (2010). Values in conflict: Youth in a culture of migration. En W. Cornelius, D. Fitzgerald, P. Lewin Fischer \& L. Muse-Orlinoff (Eds.), Mexican migration and the US economic crisis. A transnational perspective. San Diego, CA, EE.UU.: Center for Comparative Inmigration Studies/University of California San Diego. 
Herrera Carassou, R. (2006). La perspectiva teórica en el estudio de las migraciones. México: Siglo XXI.

Herrera, G. (2002). La migración vista desde el lugar de origen. Íconos. Revista de Ciencias Sociales, (15), 86-94.

Instituto Nacional de Estadística y Geografía-INEGI. (2010). XIII Censo General de Población y Vivienda 2010. México: INEGI

King, R. \& Wood, N. (2001). Media and migration: Constructions of mobility and difference. Londres, Inglaterra: Routledge.

León, G. (2008). Comunicación y migración juvenil: un imaginario de futuro en Tijuana. En M. Rebeil (Ed.), XV Anuario de la Comunicación CONEICC. México: CONEICC/Universidad Anáhuac/UIA-León/ UASLP/ITESM.

Mar, P. (2005). Unsettling potentialities: Topographies of hope in transnational migration. Journal of Intercultural Studies, 26 (4), 361-378.

Meyer, J. A. (2005). Imaginarios y migración. Poblanos en Nueva York. Revista Latina de Comunicación Social, 8 (59), 1-17.

Pacione, M. (2009). Urban geography: A global perspective. Nueva York, EE.UU.: Taylor \& Francis.

Patiño, M. (2005). Representaciones sociales, percepciones e imaginarios de jóvenes ecuatorianos artesanos indígenas y artistas estudiantes inmigrantes en Francia. Alternativas: Cuadernos de Trabajo social, (13), 165-196.

Pedone, C. (2002). Las representaciones sociales en torno a la inmigración ecuatoriana a España. Íconos. Revista de Ciencias Sociales, (14), 56-66.

Punch, S. (2007). Negotiating migrant identities: Young people in Bolivia and Argentina. Children's Geographies, 5 (1/2), 95-112.

Reguillo, R. (2009). México: contra el ábaco de lo básico. Agendas de país y desafíos para la comunicación. En J. Martín-Barbero (Ed.), Entre saberes desechables y saberes indispensables. Agendas de país desde la comunicación. Bogotá, Colombia: Centro de Competencia en Comunicación para América Latina/Friedrich Ebert Stiftung.

Revilla, J. C. (2001). La construcción discursiva de la juventud: lo general y lo particular. Papers, 63/64, 103-122. 
Rodríguez Salazar, T. (2009). Sobre el potencial teórico de las representaciones sociales en el campo de la comunicación. Comunicación y Sociedad, (11), 11-36.

Santillán, A. \& Ramírez, J. (2004). Consumos culturales urbanos: el caso de la tecnocumbia en Quito. Flacso-Ecuador, (18), 43-52.

Schimitter, B. (2008). The sociology of inmigration. En C. Brettel \& J. Holliflied (Eds.), Migration theory. Talking across disciplines. Nueva York, EE.UU.: Routledge.

Sladkova, J. (2007). Expectations and motivations of Hondurans migrating to the United States. Journal of Community \& Applied Social Psychology, 17, 187-202.

Thompson, J. B. (1998). Los media y la modernidad, una teoría de los medios de comunicación. Barcelona, España: Paidós.

Urteaga, M. (2008). Jóvenes e indios en el México contemporáneo. Revista Latinoamericana de Ciencias Sociales, Niñez y Juventud, $6(2), 667-708$.

Vega, L. (2006). Migraciones y dinámicas locales. Diferentes dinámicas locales generadas por la migración en la región de los Nonualcos. Estudios Centroamericanos, 61 (69).

Zilberg, E. (2003, 27 de mayo). La Viajera: Coming face to face with the globalization of communication networks. Documento presentado en la reunión anual de la International Communication Association, San Diego, CA, EE.UU.

Fecha de recepción: 18/08/11. Aceptación: 12/12/11. 